REVISTA DE URBANISMO

ISSN 0717-5051

http://revistaurbanismo.uchile.c
Revista de Urbanismo N40 | Junio 2019

Departamento de Urbanismo | FAU | Universidad de Chile

\title{
Experiencias de consumo como nuevos productos turísticos: la promoción de festivales y eventos en la Ciudad Autónoma de Buenos Aires
}

Consumer experiences as new tourism products: The promotion of festivals and events in the Autonomous City of

\section{Buenos Aires}

\section{Luciana Rodríguez*}

Recibido: 24 de marzo de 2019

Aceptado: 20 de junio de 2019

\section{Resumen}

Ante la premisa de competir en el mercado turístico internacional, las ciudades son promocionadas por sus gobiernos locales, ofreciendo una serie de atractivos crecientemente orientados hacia formas activas de consumo, con énfasis en recursos culturales intangibles. Lo que se define como turismo creativo. En este contexto, el marketing urbano se presenta como el instrumento dominante de la política turística, un medio para proyectar estos nuevos productos hacia sus consumidores. La ciudad de Buenos Aires no escapa a esa dinámica. Inmersa en una intensa promoción turística emprendida por su gobierno local, incorpora a la oferta turística tradicional nuevos atractivos basados en prácticas culturales, como parte del desarrollo de experiencias urbanas competitivas. A través de una metodología cualitativa, basada en la sistematización y análisis de los Festivales y Eventos ofertados a través de su página de turismo oficial, el artículo analiza cómo se capitaliza el turismo creativo para la creación de los nuevos productos turísticos en la ciudad de Buenos Aires. Los resultados obtenidos permiten constatar una tendencia creciente al turismo creativo ligada al desarrollo de productos gastronómicos y artísticos, con reducción de la intervención estatal y primacía del marketing en su desarrollo.

Palabras clave: consumo, nuevos productos turísticos, marketing urbano, política turística, promoción.

\begin{abstract}
Given the proposition of competing in the international tourism market, cities are promoted by their local governments, offering a series of attractions increasingly oriented towards active forms of consumption, with emphasis on intangible cultural resources, what is defined as creative tourism. In this context, urban marketing is presented as the dominant instrument of tourism policy, a means to launch these new products to their consumers. The city of Buenos Aires does not escape this dynamic. Immersed in an intense tourist promotion undertaken by its local government, it incorporates to the traditional tourist offer new attractions based on cultural practices, as part of the development of competitive urban experiences. Through a qualitative methodology, based on the systematization and analysis of the Festivals and Events offered through its official tourism page, the article analyzes how creative tourism is capitalized for the creation of new tourism products in the city of Buenos Aires. Aires the results obtained confirm a growing trend in creative tourism linked to the development of gastronomic and artistic products, with a reduction of state intervention and the primacy of marketing in its development.
\end{abstract}

Keywords: consumption, new tourist products, promotion, tourism policy, urban marketing.

*Filiación: CONICET- Instituto de Geografía Romualdo Ardissone, Universidad de Buenos Aires, Buenos Aires, Argentina. Contacto: arqlucianarodriguez@gmail.com

Cómo citar: Rodríguez, L. (2019). Experiencias de consumo como nuevos productos turísticos: la promoción de festivales y eventos en la Ciudad Autónoma de Buenos Aires. Revista de Urbanismo, 40, 1-16. https://doi.org/10.5354/0717$\underline{5051.2018 .52597}$ 


\section{Introducción}

Crecientemente las ciudades a nivel internacional son promocionadas como destinos turísticos por sus gobiernos locales orientándose, en general, a competir en el mercado turístico internacional. Bajo este objetivo, configuran una oferta cada vez más variada de actividades desarrolladas en el contexto urbano que, además de diversificar su repertorio de productos turísticos, actúan como insumo para la construcción de una cierta imagen de ciudad: cosmopolita, moderna, creativa, etc.

Acompañando al uso de recursos culturares tangibles, que en muchos casos caracteriza a las estrategias urbanas asociadas al turismo (activación patrimonial de edificios, creación de hitos urbanos, embellecimiento del espacio público en centros históricos, etc.), presenciamos un incremento en el uso de recursos intangibles, principalmente relacionados con experiencias de consumo en el contexto de festivales, ferias y eventos. Esto es lo que Richards (2011) ha denominado el giro creativo del turismo. Otra estrategia de los modelos de gestión urbana que buscan ajustar la imagen de sus ciudades para alinearse a los requerimientos de competitividad internacional. En este marco, entendemos que el marketing urbano se volvió el instrumento dominante de las políticas públicas con relación al turismo, en tanto contribuye a modelar y difundir esa imagen para sus consumidores.

La Ciudad Autónoma de Buenos Aires (CABA) no escapa a este fenómeno. Desde hace aproximadamente dos décadas se encuentra inmersa en una intensa promoción turística emprendida por su gobierno local, que está incorporando a su oferta más tradicional asociada al centro histórico o barrios como San Telmo y La Boca- nuevos productos urbanos: barrios étnicos, polos gastronómicos, ferias de diseño o festivales musicales. Sitios que basan su atractividad en espacios y/o prácticas culturales tradicionales como parte del desarrollo de experiencias urbanas competitivas. Consideramos que esta dinámica reciente permite examinar cómo se desarrolla el turismo creativo en la CABA, teniendo en cuenta algunas preguntas: ¿de qué manera es capitalizado para la promoción de nuevos productos turísticos? ¿Cuáles son las características de estos nuevos productos? Y, ¿cuál es el rol del sector público en su desarrollo?

Con base en estos interrogantes el artículo presenta, en su primera parte, el marco teórico que sustenta el análisis, poniendo en relación tres conceptos: el turismo creativo, la especificidad del marketing urbano y la promoción turística como política pública -en el marco de las nuevas formas de gobernanza devenidas de la neoliberalización económica-. Luego se explican estos conceptos con relación a la CABA, delineando las estrategias que el Gobierno de la Ciudad de Buenos Aires (GCBA) viene estableciendo con la actividad turística durante los últimos años. En tercer lugar, se presenta el análisis y caracterización de la oferta de Festivales y Eventos que promociona el GCBA a través de su página web oficial. Por último, se presentan las conclusiones.

\section{La promoción turística como política pública y el giro creativo}

Las iniciativas conscientes de los gobiernos por desarrollar una identidad de lugar específicamente diseñada y promoverla a segmentos de mercado se remonta al siglo XIX (Benko, 2000). Sin embargo, hace aproximadamente tres décadas -coincidiendo con el desembarco de la planificación estratégica como modelo de gestión urbana ${ }^{1}-$, se comenzó a aceptar, de forma generalizada, que la promoción de los atributos urbanos era una actividad válida para las agencias de managment del sector público y un componente esencial para la gestión de las ciudades (Kavaratzis \& Ashworth, 2005). De acuerdo con los promotores de la planificación estratégica, la proyección externa de estos atributos, a través del marketing urbano, es fundamental para el éxito del modelo, en tanto una correcta estrategia puede permitir poner en el mapa a la ciudad y crear los elementos simbólicos que permitan reconocerla con facilidad (Pascual i Esteve y De Forn y Foxá, 1999).

\footnotetext{
1 Modelo de gestión que tuvo su origen en el medio empresarial (específicamente en la Escuela de Negocios de Harvard durante los años 70), como propuesta de ajuste del ambiente interno de las corporaciones para que pudiesen enfrentar la creciente competencia en el mercado internacional -ambiente externo-, posteriormente adaptado a la esfera pública manteniendo las categorías de lectura presentes desde su origen (Novais, 2003).
} 
De esta forma, el marketing urbano irrumpió como un modelo de intervención estatal alternativo a los tradicionales centrados en el territorio, orientado, en cambio, al consumidor -residente, inversor o visitante(Calle Vaquero, 2017). Desde este modelo, la ciudad, y los lugares dentro de las ciudades, son pensados como productos susceptibles de adaptarse a los requerimientos de los consumidores.

Esto explica, en gran medida, por qué aunque dentro de la política turística -conjunto de actividades, decisiones y procesos para alcanzar objetivos con relación al turismo- los instrumentos que pueden emplearse para lograr objetivos son de índole variada, 2 es la promoción de los destinos, a través del marketing, la que cobró especial difusión y relevancia de la mano de las formas de gestión empresariales. Al respecto, Hall (2000) habla de una reducción de la intervención directa del Estado en la oferta turística, con un mayor énfasis en la colaboración público-privada. Soslayando a las políticas urbanas clásicas asociadas a planes normativos y visiones globales de la ciudad -que desde la perspectiva de la planificación estratégica se consideran obsoletas porque no permiten respuestas rápidas a las nuevas demandas del mercado-, para que la ciudad se adapte flexiblemente a las tendencias del mercado, las estrategias no vinculantes se presentan como las más adecuadas, en la medida en que reducen el control estatal (Vainer, 2000).

A su vez:

Con la desaparición de las industrias manufactureras locales y las crisis gubernamentales y financieras periódicas, la cultura se volvió más y más el negocio de las ciudades -la base de sus atractivos turísticos y su único margen competitivo-. El crecimiento del consumo cultural (de arte, comida, moda, música, turismo) y las industrias que lo abastecen son el combustible de la economía simbólica de la ciudad (...). (Zukin, 1995, p.2) ${ }^{3}$

2 Organizativos, normativos, fomento, conocimiento y comunicación (Velasco, 2016).

${ }^{3}$ Traducción de la autora.
Siguiendo a estos autores, entendemos que la cultura (y la economía simbólica en su conjunto) cumple un rol fundamental para el marketing urbano, en tanto contribuye a modelar la imagen deseada para las ciudades, además de reforzar una cierta identidad para las mismas (Kavaratzis, 2005).

Así, en el marco de la neoliberalización económica, los modelos de gobernanza que apuntan a destacar la singularidad de los lugares como medio para la diferenciación y, consecuentemente, la competitividad entre ciudades, hablan de la capitalización cultural como una de las formas más adecuadas para conseguirlo.4 Siguiendo a Harvey (1989), en la competencia por el consumo, los festivales y acontecimientos culturales se volvieron el foco de las inversiones para que las ciudades parezcan lugares innovadores, interesantes y creativos. Esto se traduce en la promoción de ciertos atributos valorados por el capital transnacional, lo que se puede enmarcar en el concepto de "ciudad como mercancía" a ser vendida, y que permitió al marketing urbano imponerse como una esfera específica dentro de la gestión y planeamiento urbano (Vainer, 2000, pp.78-79).

De esta forma asistimos, cada vez con más frecuencia, al empleo de recursos culturales para el posicionamiento de las ciudades, en donde comienza a destacar el uso de atributos intangibles por sobre los tangibles, anteponiéndose formas activas de consumo antes que las pasivas -el público como espectador- (Richards, 2011). Una dinámica que podemos enmarcar en el giro creativo del turismo: “(...) desplazamiento hacia formas activas de consumo antes que las pasivas, y un énfasis en la cultura intangible o viva en lugar del patrimonio cultural tangible, estático" (Richards, 2011, p.1237). ${ }^{5}$

Consecuentemente, autores como Richards y Palmer (2010) dirán que la distinción ya no solo se consigue contratando a arquitectos de firmas reconocidas y construyendo grandes museos, también implica la creación de ambientes animados y de sentido de lugar. Un fenómeno que podemos atribuir al rol de la experiencia para la economía actual, la respuesta de las

\footnotetext{
4 Ver, por ejemplo La Estrategia de las Ciudades (Pascual i Esteve, 1999).

5 En este artículo el autor realiza un recorrido extenso sobre las definiciones de turismo creativo.
} 
empresas al deseo de acumular experiencias por parte de los consumidores (Pine \& Gilmore, 1998). Para estos autores, la llamada economía experiencial es entendida como una nueva etapa en la evolución del valor económico, iniciada con la extracción de productos básicos (economía agraria), pasando por la producción de bienes (economía industrial) y luego la distribución de servicios (economía de servicios). Así, si los productos son fungibles, los bienes tangibles y los servicios intangibles, las experiencias deben ser memorables y ocurren en la medida en que intencionalmente se usan servicios como escenario y bienes como accesorios para atraer clientes.

Es particularmente llamativo, en este contexto, el rol que los festivales y eventos han adquirido en los últimos años como medios para el desarrollo de experiencias en el ámbito urbano. De acuerdo con Morgan (2007), para sus asistentes se trata de espacios apartados, al que acuden en búsqueda de una vivencia extraordinaria que puede tener un significado emocional y simbólico, que luego se asociará con el lugar. Otros autores afirman que su creciente difusión se ve apoyada en que "los eventos se están considerando cada vez más como plataformas para la transmisión de intangibles como valores, formación, cultura y, como no, imagen de modernidad de la ciudad" (González Reverte y Morales Pérez, 2009, p.99).

De manera creciente vemos que la oferta turística de las ciudades se compone de este tipo de productos, contribuyendo con su desarrollo a instalar una determinada imagen urbana asociada a los valores antes mencionados. Atendiendo a esta dinámica, en adelante nos proponemos explicar este proceso en la CABA, delineando la estrategia general que el GCBA ha adoptado en los últimos años con relación al turismo y la imagen de ciudad que quiere promover, a través de la oferta de festivales y eventos.

\section{Políticas orientadas al turismo en Ciudad Autónoma de Buenos Aires: un breve recorrido}

Acusadamente, y especialmente a partir de la última década, la ciudad de Buenos Aires viene siendo objeto de una fuerte campaña de promoción turística encarada por su gobierno local que, alineada con los objetivos del Gobierno Nacional, se orienta a reforzar el crecimiento del turismo receptivo y a atraer inversiones promoviendo una cierta imagen de ciudad.

Esto se ve reafirmado en los datos aportados por la Organización para la Cooperación y el Desarrollo Económicos (OCDE), donde comparativamente con los dos países de mayor peso turístico a nivel regional, México y Brasil, la economía turística en Argentina ya presentaba la mayor tasa de crecimiento en el número de turistas extranjeros hacia 2011 (Balsev Clausen y Velázquez, 2013), siendo CABA la capital regional con mayor peso dentro de su sistema nacional turístico y económico. De hecho, de acuerdo con datos aportados por la Dirección General de Estadística y Censos (Ministerio de Economía y Finanzas GCBA, s.f.a), los pernoctes de turistas extranjeros (no residentes) en la CABA se incrementaron de 11.840 .755 en el año 2005 a 17.648.981 en 2017 -con un pico de 21.427.575 en 2011-. Siendo la participación promedio de la ciudad en el total de pernoctes del país de un $62 \%$, durante el mismo período.

No obstante, el interés por desarrollar el turismo en la ciudad fue acotado hasta mediados de la década de 1990. Fue recién con la constitución de la CABA en el año $1996^{6}$ que se inicia una valoración diferente y positiva del turismo como actividad económica, cobrando aun mayor importancia después de la devaluación del año 2002 (Gómez y Zunino Singh, 2008). La reactivación económica marcada por las ventajas de la devaluación de la moneda nacional posicionó al país como un destino barato, facilitando el ingreso de divisas por parte de turistas extranjeros -el sector del turismo se convirtió en el cuarto generador de las mismas-, lo que transformó a Buenos Aires no sólo en puerta para visitar otros destinos a nivel nacional, sino en un destino en sí mismo (Zunino Singh, 2011).

\footnotetext{
${ }^{6}$ Con la reforma de la Constitución de la Nación Argentina en 1994 la Ciudad de Buenos Aires -Capital Federal-, hasta entonces regida por el gobierno nacional, pudo dictar su propia constitución y tener gobierno autónomo. Así, en el año 1996 sus ciudadanos eligieron Jefe de Gobierno, primera vez en su historia, y la ciudad adquirió el nombre de Ciudad Autónoma de Buenos Aires como lo dictó el Artículo 2 de su propia Constitución.
} 
Paralelamente a este proceso, es importante remarcar que en la década del 90, la privatización de los servicios públicos, el protagonismo de organismos internacionales de crédito y el ingreso de capitales extranjeros, fueron el contexto para que muchas de las políticas urbanas locales se alinearan al referente dominante: la ciudad como factor de competitividad en tanto sede de inversiones (Novick, 2011). No obstante, tal como indica Jajamovich (2012), la visita de los consultores catalanes también contribuyó al proceso, permitiendo que las críticas a la planificación urbana a través de la estrategia de proyecto -impugnando la estrategia del plan ligada a una visión global de la ciudad- fueran permeando en la ciudad. También es importante recordar que el retorno a la democracia dio lugar a una pluralidad de miradas sobre la ciudad, en oposición al relato totalizador y homogeneizante del período anterior, lo que derivó en múltiples proyectos urbanos de carácter parcial (Socoloff, 2007).

Así, durante 1979 y 1993, aunque especialmente con la vuelta a la democracia, aumenta el interés por las intervenciones urbanísticas españolas en relación con su propia transición democrática y -posteriormente- en relación a sus experiencias exitosas de renovación urbana, produciéndose la visita de figuras del entorno español, acompañadas de convenios de cooperación con sus ciudades (Jajamovich, 2012). De esta forma, el modelo de la planificación estratégica se afianza en la ciudad. Dio cuenta de ello el desarrollo de un Plan Estratégico en 1994 (con la posterior creación del Consejo de Planeamiento Estratégico -CoPE- en el año 1999) $^{7}$ y de un Plan Urbano Ambiental (PUA). Ambos planes, superpuestos en diversos aspectos, desarrollaron lineamentos generales acerca de la promoción y el crecimiento del turismo, por caso "promover el desarrollo de la hotelería, la gastronomía y demás servicios que conforman la infraestructura del turismo" (GCBA, 2006, p.34).

Desde este momento, el sector público comienza a desarrollar una serie de planes orientativos, aunque

\footnotetext{
7 Desde entonces, la finalidad del CoPE ha sido "elaborar planes estratégicos consensuados que ofrezcan fundamentos para la elaboración de políticas de Estado en la Ciudad Autónoma de Buenos Aires": https://www.buenosaires.gob.ar/cope/informacion-institucional
}

pocas veces específicos de turismo, que lo incluyen a partir de discursos sobre el posicionamiento y atributos competitivos de la ciudad. ${ }^{8}$ Resumidamente y a modo ilustrativo de la imagen urbana que quieren fomentar, estos planes han apuntado:

Plan Estratégico de Cultura de Buenos Aires 2001 (previo a la creación del CoPE): presta especial atención a la protección patrimonial, su reconocimiento y distinción, y se orienta al desarrollo de una imagen armoniosa de la ciudad que combine tradición y novedad; mejorar el uso de los recursos culturales locales y aprovechar la situación nacional de Buenos Aires (capital de Argentina) para crear una imagen positiva de sí misma y de la Argentina para el contexto internacional (Gómez Schettini y Troncoso, 2011).

Plan Estratégico Buenos Aires 2010: el primer plan elaborado por el CoPE lanzado en noviembre de 2004. Dentro de la línea estratégica Recuperar la ciudad como ámbito de inversión, en el documento se mencionan lineamientos para la consolidación de la competitividad turística, con miras a constituirla como una fuente regular de ingresos y empleo, ya que el turismo es concebido como generador de divisas, recursos fiscales y puestos de trabajo. En este documento el GCBA adopta el rol de facilitador de la interacción entre diferentes sectores y proveedor de los servicios de base para las actividades turísticas como infraestructura, equipamiento urbano, seguridad, promoción y desarrollo de eventos culturales. Al mismo tiempo, propone continuar con el desarrollo de la marca Buenos Aires, aunque sin especificar con qué atributos se la relaciona (CoPE, 2004).

Plan Estratégico Buenos Aires 2016: elaborado por el CoPE y publicado en 2011. Postula nuevamente el desarrollo de la marca ciudad, como el nombre de la ciudad asociado a una serie de atributos tangibles (relacionados con elementos construidos y otros propios de la condición geográfica de la ciudad); pero, principalmente, asociado a atributos intangibles como las cualidades de la población (creatividad, carácter abierto y cosmopolita, diversidad, hospitalidad y

\footnotetext{
${ }^{8}$ Como excepción, en junio de 2001 se sanciona la Ley de Turismo de la Ciudad Autónoma de Buenos Aires (Ley $N^{\circ} 600$ ), ratificando al turismo como una "actividad socioeconómica de interés público y cultural".
} 
multiculturalidad), de la economía (oportunidad de negocios y costos competitivos), del estándar de vida en la ciudad (alta calidad de vida -similar al de las ciudades más desarrolladas del mundo- y sustentabilidad) y de la cultura (gastronomía, tango y música popular, tradición gaucha, expresiones culturales y patrimonio cultural).

Cabe aclarar, además, que a partir de la primera gestión del Partido Propuesta Republicana (PRO) en 2007 se crea el Ente de Turismo de la Ciudad (ENTUR) y, con él, comienza una importante estrategia de promoción turística orientada a "consolidar el crecimiento turístico, mejorar la estructuración de los productos turísticos, aumentar el ingreso en una proporción mayor al volumen de llegadas por turismo y diversificar las áreas turísticas de la ciudad" (Rodríguez, Arqueros Mejica, Rodríguez, Gómez Schettini y Zapata, 2011, p.109). En esta línea, el GCBA lanza una serie de postulaciones internacionales (en muchos casos acompañando a declaratorias previas de Bienes Protegidos de la Ciudad en la categoría Expresiones y Manifestaciones Culturales), como la postulación del Tango Patrimonio Cultural Inmaterial de la Humanidad en 2008, el del Filete Porteño en 2015 o Buenos Aires Capital Iberoamericana de la Cultura Gastronómica (distinción otorgada por la Academia Iberoamericana de Gastronomía en 2017). ${ }^{9}$

Legitimados en estas postulaciones y en la imagen de ciudad gestada en los planes orientativos, los festivales y eventos -de carácter público y privado- adquieren especial relevancia, constituyéndose como uno de los pilares de las nuevas experiencias urbanas fomentadas para el turismo. Acusando su relevancia, en el año 2004 se decreta la creación de la Dirección General de Festivales y Eventos Centrales, dependiendo de la Secretaría de Cultura de la Ciudad (Decreto 2049/2004), con el objetivo de planificar, coordinar y organizar los festivales a desarrollarse en la CABA, acrecentando el desarrollo del turismo en la ciudad (Dirección General de Festivales y Eventos Centrales, s.f.). Incluso, de acuerdo con los datos aportados por la Dirección General de

\footnotetext{
${ }^{9}$ Con relación a esta última distinción, es importante tener en cuenta que la gastronomía "ha pasado de ser considerada una actividad subsidiaria al turismo, a una atracción en sí misma, constituyendo hoy el segmento más dinámico de la industria; un nuevo recurso para las ciudades que quieren competir en el mercado turístico internacional" (Rodriguez, 2019, p.276).
}

Estadística y Censos (Ministerio de Economía y Finanzas GCBA, s.f.b) sobre la base de datos suministrados por dicha dependencia, el número de asistentes a festivales y campeonatos organizados por el GCBA entre los años 2000 y 2017 pasó de 221.000 a 1.525.800; cifra relacionada también al paulatino incremento de la oferta durante ese período.

Promocionadas a través de las diferentes páginas web del GCBA (como la página oficial de turismo, Festivales BA o Disfrutemos BA); BA planner, herramienta on-line que organiza los atractivos que quieren visitarse en la ciudad de acuerdo a los intereses del usuario, y BA tours, un sitio web que reúne toda la oferta de actividades que se brindan desde el sector público y privado; o a través de folletería impresa entregada en puntos de información turística y en eventos específicos, estos productos se incorporan a la oferta turística oficial de la ciudad.

\section{Metodología}

Para caracterizar los nuevos productos ofertados por el GCBA, y así dar cuenta del giro creativo del turismo en CABA, la metodología de trabajo se basó un enfoque cualitativo, bajo una lógica exploratoria-explicativa, fundamentada en la sistematización y el análisis de la oferta de Festivales y Eventos promocionados a través de la página oficial de turismo del GCBA. El objeto de estudio se acotó a los 35 productos promocionados durante el mes de octubre de 2018 en el apartado Qué Hacer: www.turismo.buenosaires.gob.ar/es/agrupadornoticias/festivales-y-eventos.

A fin de tipificar la oferta, y con la voluntad de generar una metodología que pudiera extenderse al análisis de otras fuentes, los productos relevados se ordenaron de acuerdo a cinco categorías según su temática principal o el evento marco bajo el cual se promocionaban: deporte y recreación; libros y discos; arquitectura (recorridos guiados a través de edificios de la ciudad); arte y otras expresiones culturales (incluye muestras de danza, cine, música; pintura, fotografía y escultura, diseño de moda y objetos), y gastronomía. Para cada uno de los productos se recolectó información acerca de su antigüedad, periodicidad con la que se realiza, sedes de desarrollo, tipo de entrada, pertenencia o no a redes internacionales, entes organizadores, slogans de promoción y otros productos asociados. En los 
casos donde la página de turismo no mostrara esta información, se recurrió a la página Festivales $B A$ (www.festivales.buenosaires.gob.ar/es/home),

Disfrutemos BA (www.disfrutemosba.buenosaires.gob. ar), noticias de periódicos y páginas web locales, incluyéndose las páginas web propias de cada producto, en caso de tenerlas.

Finalmente, con los datos recabados se elaboró un esquema síntesis, donde las principales características de cada producto se presentaron dibujadas comparativamente y asociadas a una línea del tiempo. Esto permitió evidenciar, entre otros datos, cuáles son los productos más recientes (o nuevos) y cuáles los más antiguos (Figura 1). A su vez, se elaboró un mapa con la localización de los ítems de gastronomía y artes y otras expresiones culturales -las temáticas más cuantiosas con relación a la cantidad de productos ${ }^{10}$ a fin de mostrar las lógicas de distribución espacial a las que respondían (Figura 4).

\footnotetext{
${ }^{10}$ Se excluyeron de este mapeo las sedes de La Noche de los Museos, dado el gran volumen que representaban. Para ver un mapa con su localización referirse a: http://lanochedelosmuseos.gob.ar/2018/Inm/es/home
} 


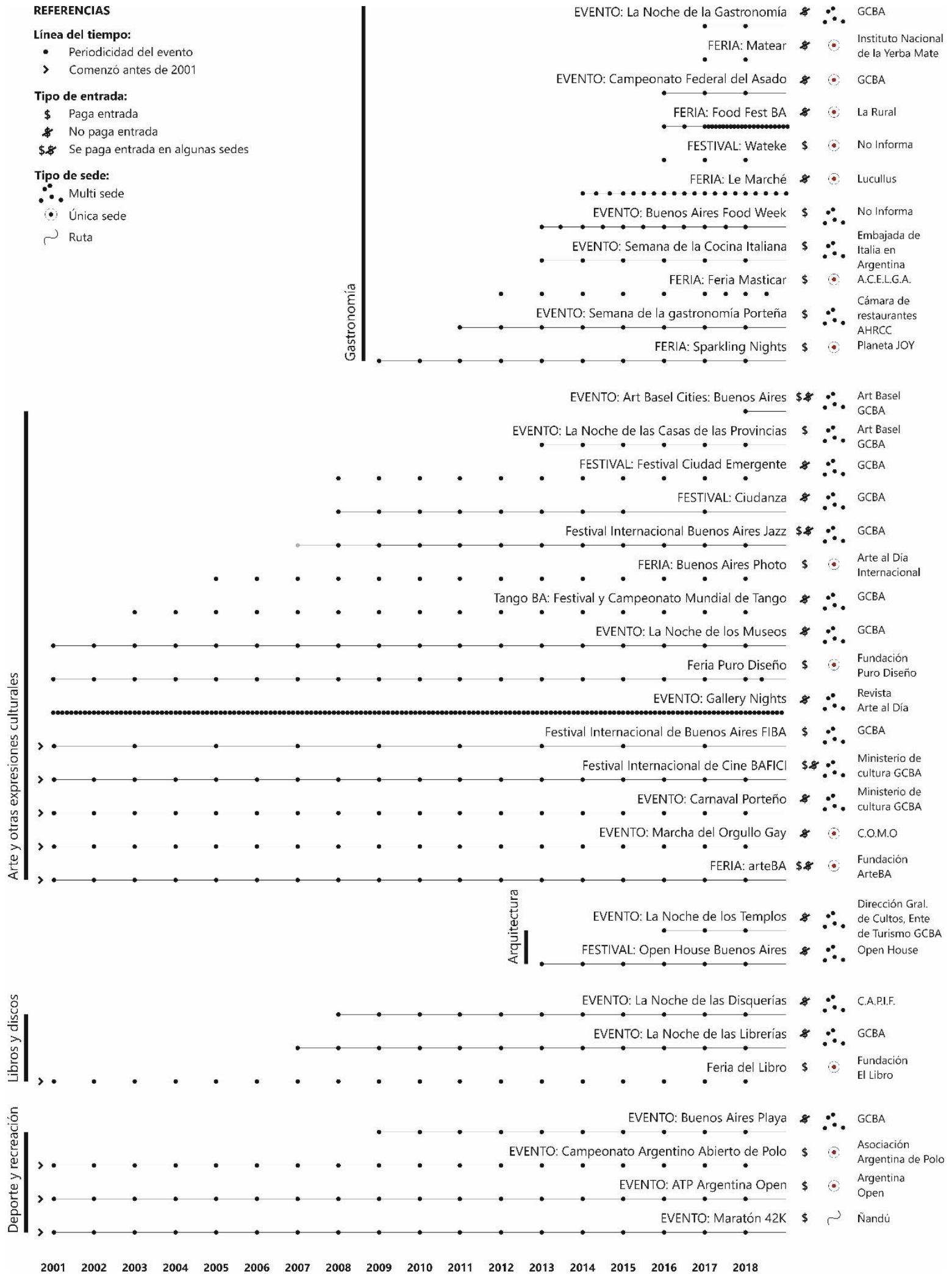

Figura 1. Festivales y Eventos ofertados del Gobierno de la Ciudad de Buenos Aires según temática. Fuente: Elaboración propia. 


\section{Resultados}

\section{Festivales, ferias y eventos: temporalidad y formas de} desarrollo

Como muestra la Figura 1, la mayor parte de las ferias y eventos promocionados comienza a desarrollarse en 2001, momento en el que el turismo cobra mayor importancia económica para la ciudad. La temática gastronomía junto con arte y otras expresiones culturales concentran la mayor parte de la oferta (once y quince productos respectivamente). El primer grupo lidera la oferta más reciente, cuyos productos se registran recién a partir de 2009; mientras que los productos de arte y otras expresiones culturales datan de la década de 1990. La oferta restante se reparte entre la temática arquitectura; libros y discos, y deporte y recreación; siendo las dos últimas las que poseen los eventos más antiguos.

Aunque estos productos se inscriben dentro de las tipologías de festivales y eventos, la categoría feria también emerge de manera recurrente, principalmente en gastronomía y arte y otras expresiones culturales. En este sentido, de acuerdo con la información empírica, se pudo identificar que la tipología festival corresponde a un tipo de evento centrado en el desarrollo de actuaciones, performances o representaciones dedicadas a alguna forma de expresión cultural y, en general, se desarrolla en diversas sedes en simultáneo. Las ferias se relacionan a eventos focalizados en la actividad comercial, donde se exponen y venden productos o servicios en recintos únicos. Mientras que en la categoría general de eventos, entendidos como acontecimientos esporádicos desarrollados durante un período de tiempo acotado, se inscriben todos los productos relacionados con el deporte y recreación, campeonatos o competencias, y todos aquellos titulados a partir de su desarrollo temporal (Noche de..., Semana de..., etc.).

A su vez, vinculados al tipo y cantidad de sedes de desarrollo, los productos relevados se pueden caracterizar según dos modalidades: aquellos basados en la visita a sitios o recintos puntuales, principalmente asociados a eventos deportivos o ferias, ${ }^{11}$ o aquellos que ofrecen recorridos o itinerarios basados en la proximidad temática o geográfica de sus sedes. En este último caso, ya sea a través de recorridos guiados, como los que ofrecen el Open House Buenos Aires y La Noche de los Templos, a través de circuitos sugeridos como el agrupamiento de restaurantes temáticos de La Semana de la Cocina Italiana, o por criterios de proximidad, como las galerías de la Gallery Nights, se ofrece al público la posibilidad no sólo de consumir una experiencia puntual, sino también de abarcar múltiples experiencias dentro de un recorrido significativo. Lo que a su vez se transforma en un producto en sí mismo.

Al indagar en el origen de estos productos, podemos ver que muchos fueron multiplicando sus sedes $y$ colonizando nuevos sectores de la ciudad a través del tiempo. Esto se corrobora principalmente en los eventos gastronómicos basados en la oferta de restaurantes con menú promocional como La Noche de la Gastronomía o Buenos Aires Food Week, y en los productos artísticos BAFICI, FIBA, el Festival y Campeonato Mundial de Tango y el Festival Internacional de Jazz, que han incorporado espacios de carácter secundario en Barrio 31, Barrio 1.11.14 o Villa 20-21 (Figura 2).

${ }^{11}$ El tradicional predio ferial de La Rural se repite de manera recurrente en la categoría ferias. Fundado en 1878, se ubica en el barrio de Palermo y es dirigido por la Sociedad Rural Argentina (asociación civil). 
POPULaR provincia politica policiales deportes espectaculos teconos

\section{Se realiza el $11^{\circ}$ Festival Internacional de Buenos Aires (FIBA)}

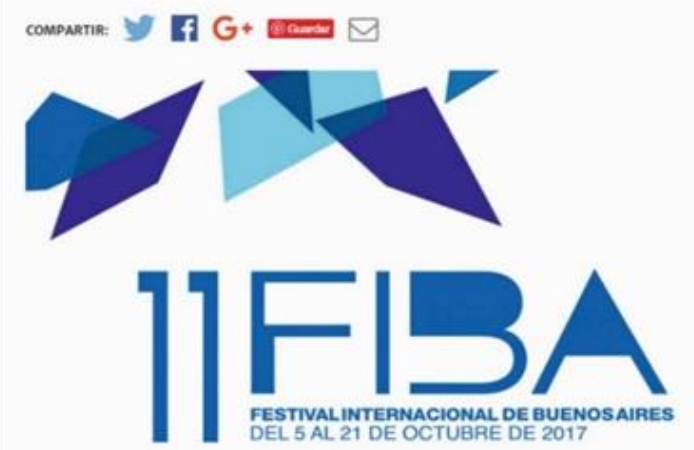

Por primera vez la programación del evento llegará al Barrio 31 de Retiro, al Barrio Ramón Carrillo, al Barrio Fátima de Villa Soldati y al Barrio villa 20 de Villa Lugano, tanto con producciones nacionales como internacionales.

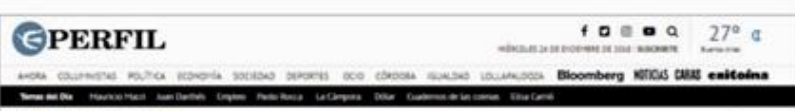

Por primera vez, el Bafici llegó a las villas porteñas

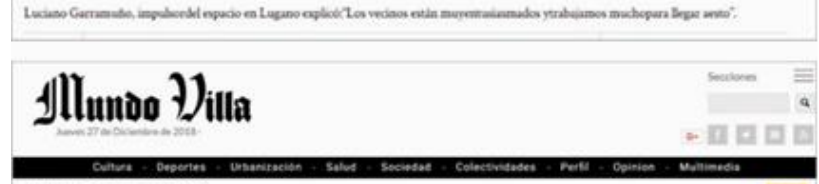

El Festival Buenos Aires Jazz Ilega a Cildañez y Villa 20

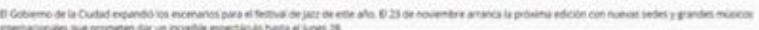

Figura 2. Nuevas sedes para los festivales y eventos artísticos. Fuentes: https://www.diariopopular.com.ar/, https://www.perfil.com/, https://mundovilla.com/archive/

Sin embargo, una breve comparación entre ambos grupos temáticos permite identificar dos lógicas diferentes de distribución espacial: los productos gastronómicos se ubican, por lo general, en la zonas centro y norte de la ciudad en relación a los polos gastronómicos (Palermo, Las Cañitas, Puerto Madero, Recoleta, San Telmo); ${ }^{12}$ mientras que los productos artísticos, localizados principalmente en el centro y sureste de la ciudad (en coincidencia con el Distrito de las

12 Ver Polos Gastronómicos en:

https://turismo.buenosaires.gob.ar/es/article/polosgastron\%C3\%B3micos
Artes que comprende los barrios de La Boca, San Telmo y Barracas) comienzan a penetrar también en el suroeste, en relación al Distrito del Deporte (

La oferta relevada presenta, además, diferencias asociadas al grado de participación del público. En un extremo, registramos productos donde el visitante se involucra activamente y es parte de la construcción de la actividad central, como La Maratón $42 K$ o el Buenos Aires Playa; eventos que demandan la participación del público para su desarrollo. En el otro, aquellos basados en la contemplación de una atracción central por parte de los asistentes -participación pasiva-, como el Campeonato Abierto de Polo o el Campeonato Mundial de Tango. En el medio, podemos ubicar la mayor parte de la oferta basada en la contemplación de espectáculos de pequeña escala y en el consumo activo de productos y servicios por parte del público (principalmente gastronómicos o de diseño a través de múltiples stands dedicados a ello), siendo la sumatoria de todos estos aspectos la experiencia que se promociona. No obstante, incluso tratándose de eventos basados en la contemplación de una actividad central, la mayoría de los productos relevados son acompañados de shows artísticos, competencias, talleres, puestos gastronómicos, etc. De esta manera, aunque en general no exigen el pago de entrada, estas actividades obligan a alguna forma de consumo pago para la permanencia.

La mayor parte de los productos relevados se desarrollan con una frecuencia anual. Sin embargo, aquellos que comenzaron teniendo una frecuencia mayor, como la Food Fest BA y Le Marché, la incrementaron aún más con el paso del tiempo. A su vez, la Feria Masticar, desarrollada anualmente, incorporó una segunda edición en 2017, acusando el éxito de este tipo de producto.

\footnotetext{
${ }^{13}$ Los distritos económicos de la CABA (Tecnológico, Audiovisual, de Diseño, de las Artes y del Deporte) son delimitaciones administrativas generadas con el objetivo de incentivar la radicación de empresas de determinados rubros en zonas con bajo desarrollo relativo, a través de exenciones fiscales e inversión pública.
} 


\section{Re-empaquetamiento y el rol del sector público en la promoción}

La capacidad de estos productos de ser vendidos a múltiples públicos, e incluso de adoptar diferentes categorías, es una cualidad ampliamente explotada para la promoción de los Festivales y Eventos; un aspecto importante para entender que la creciente diversidad en la oferta no está necesariamente relacionada con la cantidad, sino con una multiplicidad de discursos que nombran y vuelven a nombrar a los mismos lugares bajo diferentes atributos.

En este sentido, encontramos que mucho de los productos relevados son promocionados por el sector público en el marco de diversos slogans o programas que actúan como aglutinadores de festivales, ferias y eventos con características compartidas. Nos referimos, por ejemplo, a Las Noches de Buenos Aires, Disfrutemos BA, Festivales BA, BA Vamos Buenos Aires o Buenos Aires Capital Gastronómica. Frecuentemente mencionados como programas, aunque careciendo del desarrollo normativo para constituirse como tales,14 en muchos casos actúan como marco de una determinada oferta temática a través de páginas web específicamente diseñadas para la promoción y acompañados de una serie de logos e imágenes que contribuyen a reforzar la identidad visual que se quiere difundir (Figura 3).

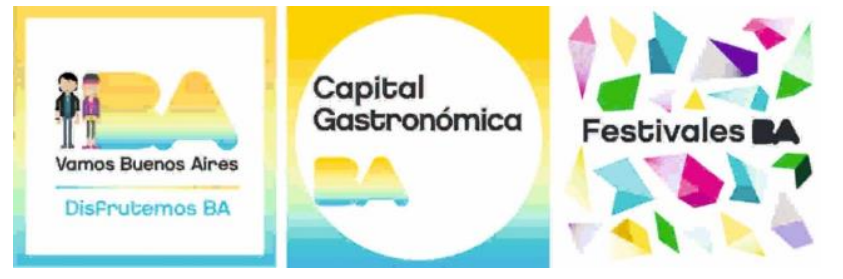

Figura 3. Slogans y programas del GCBA. Fuentes: https://www.facebook.com/GCBA/

https://twitter.com/ba_gastronomica, https://www.facebook.com/FestivalesGCBA/

\footnotetext{
${ }^{14}$ En las diferentes noticias relevadas se registró esta denominación, no obstante, no se encuentran registros oficiales de cómo operan estos programas.
}

Por caso, en el marco de Las Noches de Buenos Aires se promocionan: La Noche de las Librerías, La Noche de las Disquerías, La Noche de la Gastronomía, La Noche de las Provincias, La Noche de los Museos y La Noche de los Templos. Se trata de eventos basados en circuitos a pie, a través de los cuales el público puede recorrer las diferentes sedes involucradas durante un horario de apertura excepcional o extendido. Pero, a su vez, algunos de esos mismos productos son promocionados bajo el slogan Disfrutemos BA, una agenda cultural materializada en la página web que agrupa la oferta de eventos de la CABA, o BA Capital Gastronómica, común denominador de los productos gastronómicos ofertados y promovido a través de cuentas de Twitter, Instagram y Facebook. Otro ejemplo es Festivales BA, página web específica que reúne los festivales ofertados por el GCBA vinculados al arte $\mathrm{y}$ otras expresiones culturales pero que, muchas veces, también promocionados bajo el slogan BA Vamos Buenos Aires.

A su vez, si bien la pertenencia de estos productos a redes internacionales, o la réplica del formato de eventos desarrollados en otras ciudades, no predominan en la oferta, sí se verifica en algunos casos -especialmente en el grupo arte $y$ otras expresiones culturales-. La pertenencia a estas redes también actúa como una forma de empaquetamiento a escala internacional, conectando el producto local con sus pares en otras ciudades. Por ejemplo la Gallery Nights tiene sus ediciones especiales de verano en la ciudad de Punta del Este, Uruguay.

Otro aspecto de este tema surge al analizar las sedes de desarrollo de los diferentes productos, donde encontramos que muchos nombres se repiten. Por caso, ya sea que se trate del establecimiento en su localización original, o a través del formato foodtruck dentro de los predios de los festivales y ferias, ciertos nombres de restaurantes suelen reiterarse en los diferentes eventos. Esto pone en relieve cierta tendencia a ofertar los mismos lugares bajo diferentes atributos: por ejemplo, un mismo establecimiento puede ofrecer su menú como comida italiana, comida tradicional o comida casera. Incluso eventos específicos como Matear, tienen su propio stand dentro de otros eventos como en el Campeonato Federal del Asado. Algo similar sucede para el caso de Art Basel, que además de las exposiciones e instalaciones específicas del evento, incluye los 
productos Gallery Weekend y Buenos Aires Photo, promocionados también de forma independiente.

Finalmente, una cuestión clave en la tipificación de estos productos se relaciona con sus promotores. Aunque todos son ofertados a través de la página oficial de turismo del GCBA, los festivales, ferias y eventos pueden agruparse, por un lado, entre aquellos promovidos por el sector privado, en donde el GBCA es mencionado como sponsor, presentador $O$ aval institucional -figura muchas veces compartida con empresas privadas-. Por otro lado, aquellos directamente promovidos por el GCBA o alguna de sus dependencias (como la Dirección General de Promoción Cultural), mayormente vinculados a la temática arte $y$ otras expresiones culturales. En ambos casos, es difícil determinar cuáles son los límites o alcances del sector público en el desarrollo de los productos turísticos. Por ejemplo, el análisis de notas periodísticas recientes sobre cómo opera la promoción del Buenos Aires Playa permitió ver que en la edición 2018, la organización del evento estuvo concesionada a la empresa Prado del Ganso S.A., mientras que para la edición de 2019 estará a cargo de la empresa Marketing Dimension S.A. ${ }^{15}$

15 Ver ¿Cuánto costará Buenos Aires Playa 2018? en https://www.nueva-ciudad.com.ar/notas/201801/35803-cuantocostara-ba-playa.html y Buenos Aires Playa: la estafa de Larreta que costará \$42 millones y no tendrá ni una gota de agua en https://www.diarioregistrado.com/politica/buenos-aires-playa--la-

estafa-de-larreta-que-costara--42-millones-y-no-tendra-ni-una-gota-deagua a5c2a0d27e734eb631725c477 


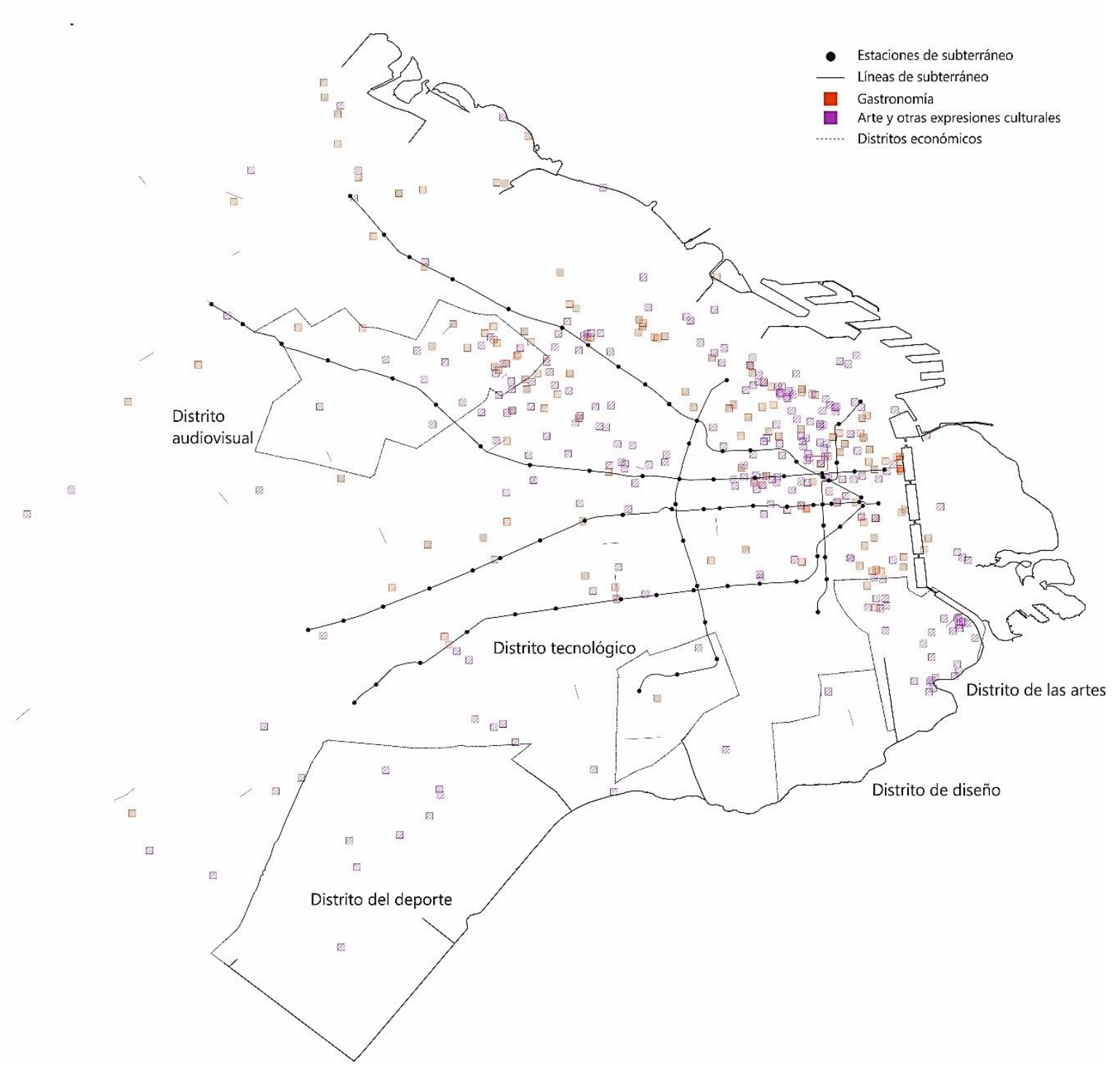

Figura 4. Localización de los productos de gastronomía y arte y otras expresiones culturales. Fuente: elaboración propia.

\section{Conclusiones}

Este artículo se propuso analizar el desarrollo del turismo creativo en la CABA, a través de la promoción de los Festivales y Eventos del GCBA. A partir de los resultados obtenidos, es posible confirmar, en primer lugar, una tendencia creciente al turismo creativo en la ciudad de Buenos Aires.
El empleo de formas activas de consumo con énfasis en la cultura intangible (Richards, 2011) puede constatarse, por un lado, a partir del incremento general, en el desarrollo de festivales, ferias y eventos basados en prácticas culturales locales como Matear, el Campeonato Federal del Asado, el Campeonato Mundial de Tango o el Carnaval Porteño. Incluso, acusa su importancia para la ciudad, la creación de un ente específico dentro del GCBA destinado a su desarrollo y promoción: la Dirección 
General de Festivales y Eventos Centrales, dependiente de la Secretaría de Cultura de la ciudad.

Por otro lado, la combinación de espectáculos con diferentes modalidades comerciales -venta de bienes y servicios, especialmente gastronómicos-, implican la participación activa de sus asistentes, principalmente, a través del consumo pago. Este aspecto, sumado al desarrollo de eventos gastronómicos a partir de 2009, confirma la relevancia que el segmento adquirió en el último tiempo. Sirviendo a los fines del turismo creativo, permite la puesta en valor de experiencias culinarias e implica, necesariamente, la participación activa de su público. Incluso, emergiendo del análisis empírico, la categoría feria como un espacio específico de compra y venta de productos, asociados principalmente a la comida, da cuenta de la importancia de este aspecto.

En segundo lugar, y en el marco del modelo empresarial que caracteriza a la actual gestión de la ciudad, el análisis también demostró una inclinación a la institucionalización (incorporar a la oferta oficial) de productos desarrollados por el sector privado. En eso casos, el GCBA actúa como agente de promoción a través de canales oficiales, presentándose como apoyo institucional o sponsor, en la mayoría de los casos, roles compartidos con empresas privadas. Además, no se especifica cuáles son las obligaciones de esas figuras ni los beneficios generados para el sector público por la promoción. Esto permite alinear al caso local con el énfasis en la colaboración público-privada y la reducción de la intervención directa estatal en la oferta turística explicada por Hall (2000).

Los resultados dan cuenta, a su vez, de la importancia del marketing en las políticas de turismo, como un medio para adaptar la oferta a los requerimientos de los consumidores, consolidando una cierta imagen de ciudad. En este caso, una ciudad con variada oferta de eventos singulares ligados a la tradición local (como se comentó antes) o alternativos. Pero, también, conectada con tendencias globales a partir de su vinculación con redes de ciudades y eventos internacionales.

No obstante, la especificidad del caso nos demuestra que el marketing no sólo sirve para proyectar imagen de ciudad, sino también, imagen de gobierno. A simple vista, el impacto visual generado a través de logos, banners y slogans asociados a la actual gestión parece consolidar la imagen del GCBA como impulsor directo de una variada oferta de actividades en toda la ciudad. Sin embargo, cabe cuestionarse a quienes realmente se dirige esa oferta, en su mayoría, ligada a las industrias de la economía simbólica.

Finalmente, la promoción de los productos analizados parece alinearse a dinámicas de valorización económica de ciertos sectores de la ciudad, aunque adoptando temáticas particulares según la localización. Impulsados desde el sector privado, la localización de los productos gastronómicos refuerza la consolidación de los polos temáticos en los sectores de mayor valorización inmobiliaria de la ciudad. Mientras que los productos agrupados en arte y otras expresiones culturales, impulsados por el sector público, penetran en el suroeste de la ciudad (en coincidencia, por ejemplo, con la estrategia de valorización promovida por el Distrito del Deporte), aunque con sedes secundarias [R]

\section{Referencias}

Balsev Clausen, H. y Velázquez, M. (2013). El turismo cultural en Argentina, México y Brasil. Avances y desafíos. Diálogos Latinoamericanos, 21, 9-30.

Benko, G. (2000). Estrategias de comunicación y marketing urbano. Revista Eure, 26(79), 67-76. https://doi.org/10.4067/s0250-71612000007900004
Calle Vaquero, M. (2017). Incidencias económicas y funcionales del turismo en las ciudades históricas (Tesis doctoral). Universidad Complutense de Madrid, Facultad de Geografía e Historia. 
Consejo de Planeamiento Estratégico. (2011). Plan Estratégico Buenos Aires 2016, con perspectiva metropolitana. Ciudad Autónoma de Buenos Aires. Disponible en

https://www.buenosaires.gob.ar/cope/basedocumental/planes-estrategicos-comparados

Consejo de Planeamiento Estratégico. (2004). Plan Estratégico Buenos Aires 2010. Ciudad Autónoma de Buenos Aires.

Consejo de Planificación Estratégica (s.f.). Información Institucional. Disponible en https://www.buenosaires.gob.ar/cope/informacioninstitucional

Dirección General de Festivales y Eventos Centrales (s.f.). Dirección General Festivales y Eventos Centrales. Disponible en https://www.buenosaires.gob.ar/cultura/institucional -subsecretaria-gestion-cultural/dg-festivales-yeventos-centrales

GCBA. (2006). Documento Plan Urbano Ambiental. CABA: Gobierno de la Ciudad de Buenos Aires. Disponible en http://www.cedet.edu.ar/archivos/Bibliotecas Archiv os/PUA\%20CABA\%2006.pdf

Gómez Schettini, M. y Troncoso, C. (2011). Tourism and cultural identity: Promoting Buenos Aires as the cultural capital of Latin America. Catalan Journal of Communication \& Cultural Studies, 3(2), 195-209. https://doi.org/10.1386/cjcs.3.2.195 1

Gómez, M. y Zunino Singh, D. (2008). La (re) valorización de la zona sur y su patrimonio histórico cultural como recurso turístico. En Hilda Herzer (Comp.) Barrios al Sur. Renovación y pobreza en la ciudad de Buenos Aires. Argentina: Café de las Ciudades.

González Reverte, F. y Morales Pérez, S. (2009). Ciudades efímeras: Transformando el turismo urbano a través de la producción de eventos. Barcelona: Universitat Oberta de Catalunya.

Hall, M. (2000). Tourism Planning. Policies, processes and relationships. Essexs: Prentice Hall.

Harvey, D. (1989). From Managerialism to Entrepreneurialism: The Transformation in Urban Governance in Late Capitalism. Geografiska Annaler, 71(1), 3-17. https://doi.org/10.2307/490503
Jajamovich, G. (2012). Intercambios internacionales, estrategias urbanísticas y aspectos políticos: España, Buenos Aires y Rosario (1979-1993). Revista Iberoamericana de Urbanismo, 7, 19-30.

Kavaratzis, M. \& Ashworth, G. J. (2005). City Branding: an effective assertion of identity or a transitory marketing trick? Tijdschrift voor Economische en Sociale Geografie, 96(5), 506-514. https://doi.org/10.1111/j.1467-9663.2005.00482.x

Kavaratzis, M. (2005). Place Branding: A Review of Trends and Conceptual Models. The Marketing Review, 5(4), 329-342.

https://doi.org/10.1362/146934705775186854

Ministerio de Economía y Finanzas GCBA (s.f.a). Pernoctaciones de turistas no residentes por destino. Aeropuerto Internacional de Ezeiza y Aeroparque Jorge Newbery. Años 2005/4to. trimestre 2018. Disponible en: https://www.estadisticaciudad.gob.ar/eyc/?cat=142

Ministerio de Economía y Finanzas GCBA (s.f.b). Asistentes a festivales y campeonatos organizados por el GCBA por festival o campeonato. Ciudad de Buenos Aires. Años 2000/2017. Disponible en https://www.estadisticaciudad.gob.ar/eyc/?p=27492

Morgan, M. (2007). Festival spaces and the visitor experience. En M. Casado-Diaz; S. Everett y J. Wilson (Eds.) Social and Cultural Change: Making Space(s) for Leisure and Tourism (pp. 113-130). Reino Unido: Lesiure Studies Association.

Novais, P. (2003). Uma estratégia chamada "Planejamento estratégico". Deslocamentos espaciais e atribuições de sentidos na teoría do planejamento urbano (Tesis Doctoral). Universidad Federal de Río de Janeiro.

Novick, A. (2011). Planes, Ideas y Proyectos para el AMBA. Buenos Aires: Consejo Profesional de Arquitectura y Urbanismo.

Pascual i Esteve, J. M. y De Forn y Foxá, M. (1999). La estrategia de las ciudades. Los Planes Estratégico como instrumento: Métodos, técnicas y buenas prácticas. Barcelona: CIDEU. 
Pine, B. J. \& Gilmore, J. H. (1998). Welcome to the Experience Economy. Harvard Bussines Review. Disponible en https://hbr.org/1998/07/welcome-tothe-experience-economy

Richards, G. (2011). Creativity and tourism. The State of the Art. Annals of Tourism Research, 38(4), 12251253.

Richards, G. y Palmer, R. (2010). Eventful Cities: Cultural Management and Urban Revitalisation. Oxford: Elsevier.

Rodriguez, L. (2019). Palermo Viejo: Palermo Soho. Los nuevos espacios de consumo como insumo del turismo urbano en Buenos Aires. C. Milano y J. Mansilla (Coords.), Ciudad de vacaciones. Conflictos urbanos en espacios turísticos, (pp. 289-326). Barcelona: OACU-Pol.len.

Rodríguez, M., Arqueros Mejica, S., Rodríguez, F., Gómez Schettini, M. y Zapata, M. (2011). La política urbana 'pro': continuidades y cambios en contextos de renovación en la Ciudad de Buenos Aires. Cuaderno Urbano: espacio, cultura y sociedad, 11(11), 101-121.

Socoloff, I. (2007). Buenos Aires bajo el lente de la Planificación Estratégica. Discursos sobre la ciudad presentes en el Plan Estratégico Buenos Aires 2010. En $4^{\circ}$ Jornadas de jóvenes investigadores, Instituto de Investigaciones Gino Germani. Buenos Aires, Argentina.
Vainer, C. (2000). Pátria, empresa e mercadoria. Notas sobre a estratégia discursiva do planejamento estratégico. En O. Arantes, C. Vainer y E. Maricato. A cidade do pensamento único. Desmanchando consensos (pp. 75-104). Petrópolis: Vozes.

Velasco, M. (2016). Entre el poder y la racionalidad: gobierno del turismo, política turística, planificación turística y gestión pública del turismo. PASOS, Revista de Turismo y Patrimonio Cultural, 14(3), 577-594. https://doi.org/10.25145/j.pasos.2016.14.038

Zukin, R. (1995). The Cultures of Cities. Nueva York: Christie's.

Zunino Singh, D. (2011). Los usos económicos de la cultura en los procesos de renovación urbana. Las políticas de patrimonio y el turismo en el caso del barrio de San Telmo (Casco Histórico de la ciudad de Buenos Aires) (Tesis de Maestría). Maestría en Sociología de la Cultura y el Análisis Cultural. IDAESUNSAM. 